\title{
A condition-specific codon optimization approach for improved heterologous gene expression in Saccharomyces cerevisiae
}

\author{
Amanda M Lanza ${ }^{1,3}$, Kathleen A Curran ${ }^{1}$, Lindsey G Rey ${ }^{1}$ and Hal S Alper ${ }^{1,2^{*}}$
}

\begin{abstract}
Background: Heterologous gene expression is an important tool for synthetic biology that enables metabolic engineering and the production of non-natural biologics in a variety of host organisms. The translational efficiency of heterologous genes can often be improved by optimizing synonymous codon usage to better match the host organism. However, traditional approaches for optimization neglect to take into account many factors known to influence synonymous codon distributions.
\end{abstract}

Results: Here we define an alternative approach for codon optimization that utilizes systems level information and codon context for the condition under which heterologous genes are being expressed. Furthermore, we utilize a probabilistic algorithm to generate multiple variants of a given gene. We demonstrate improved translational efficiency using this condition-specific codon optimization approach with two heterologous genes, the fluorescent protein-encoding eGFP and the catechol 1,2-dioxygenase gene CatA, expressed in S. cerevisiae. For the latter case, optimization for stationary phase production resulted in nearly 2.9-fold improvements over commercial gene optimization algorithms.

Conclusions: Codon optimization is now often a standard tool for protein expression, and while a variety of tools and approaches have been developed, they do not guarantee improved performance for all hosts of applications. Here, we suggest an alternative method for condition-specific codon optimization and demonstrate its utility in Saccharomyces cerevisiae as a proof of concept. However, this technique should be applicable to any organism for which gene expression data can be generated and is thus of potential interest for a variety of applications in metabolic and cellular engineering.

Keywords: Codon optimization, Codon bias, Saccharomyces cerevisiae, Heterologous expression, Synthetic biology

\section{Background}

Codon optimization is commonly used to improve heterologous gene expression, especially in the context of synthetic biology and metabolic and cellular engineering [1,2]. While most commonly employed in prokaryotic systems [3-5], codon optimization has also been described in eukaryotic systems such as yeast $[1,6-8]$. The basic premise behind this approach is that the distribution of the 64 unique DNA codons is non-random. Specifically, the occurrence of

\footnotetext{
* Correspondence: halper@che.utexas.edu

'Department of Chemical Engineering, The University of Texas at Austin, 200

E Dean Keeton St. Stop C0400, Austin, TX 78712, USA

${ }^{2}$ Institute for Cellular and Molecular Biology, The University of Texas at

Austin, 2500 Speedway Avenue, Austin, TX 78712, USA

Full list of author information is available at the end of the article
}

synonymous codons (i.e. different codons all encoding for the same amino acid) within any genome is not uniform, resulting in both rare and abundant codons. The distribution of preferred codons varies across all organisms $[1,9,10]$ giving rise to a host-specific codon usage bias (CUB) [11]. Codon usage, especially the high prevalence of rare codons, is known to influence translational efficiency [12]. As a result, the most common strategy for codon optimization is to replace rare codons with more frequently occurring ones, thereby matching the CUB of the host organism.

Typically, the CUB for a given organism is determined using the Codon Usage Tabulated from GenBank (CUTG) [13]. This process calculates the frequency of codon usage across all annotated protein coding genes and is the 
primary dataset used for codon optimization. Several alternative codon usage tables exist including the codon adaptation index (CAI) $[14,15]$, codon bias index (CBI) [16] and effective number of codons $\left(\mathrm{N}_{\mathrm{c}}\right)$ [17]. As an outgrowth of these approaches, several online optimization programs have been developed and are freely available [18-20]. Despite the promise of these approaches, much of this research has focused on studying and describing endogenous gene expression. Moreover, traditional codon optimization does not always lead to improved expression compared to a wild-type, unmodified sequence [21-23]. In fact, in a survey of 44 synthetic genes manufactured by Blue Heron Biotechnology (http://www.blueheronbio.com/ assets/documents/BlueHeronBioExpressionSurvey.pdf), $32 \%$ of the "optimized" synthetic genes expressed at lower levels than the wild-type. Thus, alternative strategies are required to further improve codon optimization for heterologous genes, especially in eukaryotic hosts and for biotechnological applications.

We posit that the limitations to traditional codon optimization stem from the fact that traditional methods for CUB calculations utilize all potential coding regions of the genome. It is well documented that tRNA abundance is influenced by changes in environmental factors including growth condition and cell-cycle [24-27]. Moreover, much of an organism's protein coding genes are lowly expressed and thus minimal evolutionary pressure has been present to drive efficient natural evolution and optimization $[11,28]$. As a result, it is expected that the effective CUB for a given growth condition may differ from the whole-genome based CUB. Thus, a traditional codon optimization approach neglects cell conditions and considers all of a genome's protein coding information as equal. As a result, these approaches fail to capture important nuances of the effective CUB that may be essential for guaranteeing expression of a heterologous gene. In addition, traditional approaches neglect evidence that adjacent codons co-evolve [29-31]. Finally, it is not always wise to consistently utilize abundant codons, especially in biotechnological applications where multiple genes are optimized and the cognate tRNA may eventually become limiting [27].

Here we demonstrate that a CUB generated using only genes expressed under a given condition can enable improved codon optimization in Saccharomyces cerevisiae compared to a CUB generated using the CUTG. We refer to this alternative approach as 'condition-specific codon optimization.' Furthermore, we utilize a probabilistic method that incorporates codon context into optimized gene design and thus results in multiple variants to be tested rather than a singular design. We demonstrate the utility of this technique in $S$. cerevisiae through the condition-specific codon optimization of two heterologous genes: a green fluorescent protein variant originally optimized for expression in Escherichia coli, and the catechol 1,2-dioxygenase enzyme from Acinetobacter baylyi. To do so, we created a CUB for two specific conditions of interest: constitutive high expression and high expression in stationary phase growth. The resulting optimized genes yielded more protein activity under the conditions for which they were optimized. Furthermore, our best catechol 1,2-dioxygenase gene variant resulted in 2.9-fold higher activity than a commercially optimized gene variant. This technique should be applicable to any organism for which gene expression data can be generated and is thus of potential interest for a variety of applications in metabolic and cellular engineering.

\section{Results and discussion}

Developing a condition-specific codon usage bias matrix

To establish condition-specific codon optimization, we developed a simple workflow for optimizing heterologous genes (outlined in Figure 1a).

First, the condition under which the heterologous gene of interest will be expressed must be identified and genome-scale expression data for the host should be obtained under this condition. While we have selected DNA microarray data for this study, it is conceivable to use additional sources such as RNA-seq or proteomics. For commonly studied organisms, the desired information is publically available in databases such as Gene Expression Omnibus (GEO), the Center for Information Biology Gene Expression database (CIBEX) and Array Express.

Second, using the appropriate dataset, genes that are differentially up-regulated or highly expressed under the desired condition are identified. From this set of genes and their corresponding DNA sequences, codon frequency and probability can be determined for codon pair usage (codon context). The python script used to generate the condition-specific tables and matrices is entitled 'CodonUsageAnalysis' (see Additional file 1). The output of this analysis is a 61 by 61 matrix termed the 'codon bias matrix' (the three stop codons were excluded from this matrix). We chose to focus on codon context rather than individual codons as previous studies suggest that codon context may be more important for gene optimization [29-32] and that this context directly correlates with translation elongation rate [33]. In particular, steric hindrance of charged tRNAs by adjacent codons can be avoided by considering the impact of adjacent codon pairing [30].

Third, the condition-specific matrix can be used to reconstruct a series of codon optimized genes that would follow the codon bias rules of the condition of interest. By considering each adjacent codon pairing in the chain, we probabilistically reconstruct the DNA sequence from the protein sequence utilizing the codon 

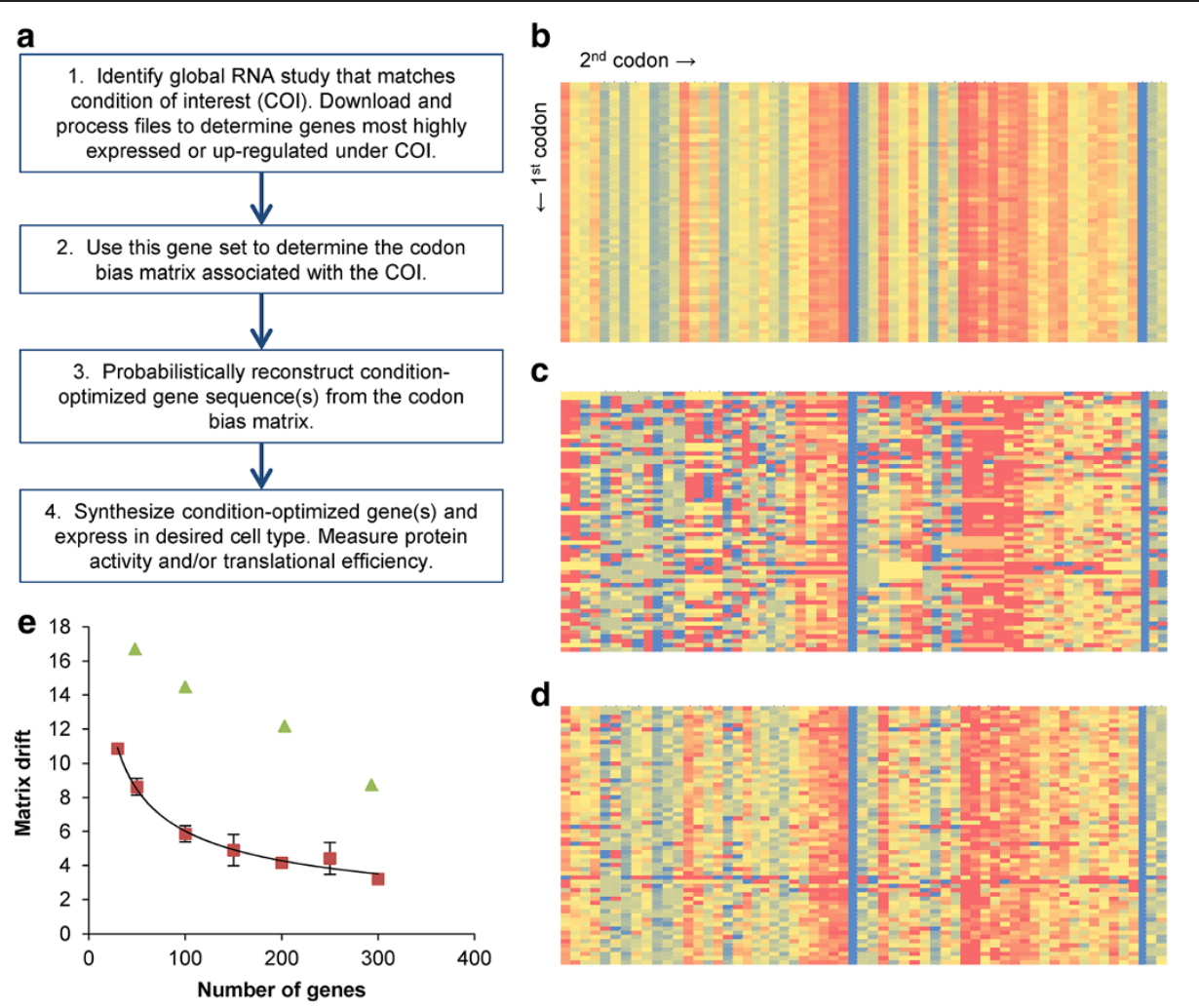

Figure 1 Condition-specific codon optimization utilizing systems level information and codon context. a. A generic workflow to enable a condition-specific codon optimization algorithm in any organism from gene expression data. $\mathbf{b}$. The control codon matrix is compiled from all 6,666 protein-coding genes in S. cerevisiae and serves as a point of comparison for condition-specific matrices. The first amino acid is indicated by the first column, and the second amino acid by the first row. The color indicates probability between 0 (red) and 1 (blue). c. The high expression codon optimization matrix is compiled from the 100 most highly expressed protein-coding genes in S. cerevisiae [35]. $\mathbf{d}$. The stationary phase codon optimization matrix is compiled from the 50 most highly expressed protein-coding genes in S. cerevisiae grown for 3 days, compared to an exponential population [38]. e. The matrix drift from the control matrix (as indicated by Frobenius matrix norm) versus number of genes used to generate the codon usage matrices was plotted for codon usage matrices generated from a random sampling of genes (red squares) and the most highly expressed genes [35] (green triangles). The random data sets were fit with a power regression model. Standard deviations from five independent samples were used to generate error bars.

context probabilities stored in the condition-specific matrix (performed via a python script entitled 'GeneDesign' included as Additional file 2). As an example, if the first two amino acids of the protein sequence are methionine followed by cysteine, there are two possible corresponding DNA sequences: ATGTGT and ATGTGC. The condition-specific matrix stores the probability in which each pairing occurred in the set of up-regulated genes for a given condition. GeneDesign assigns a DNA sequence based on the corresponding probability. For example, if $60 \%$ of Met-Cys pairs are ATGTGT and only $40 \%$ are ATGTGC, GeneDesign will probabilistically select ATG TGT $60 \%$ of the time and ATGTGC $40 \%$ of the time for each occasion that a Met-Cys pair is present in the peptide sequence. As a result of the probabilistic design, several variants of codon optimized genes are generated. Moreover, this process also ensures a balance in codon usage rather than the exclusive use of specific codons which can result in bottlenecks with the formation of charged
tRNA-amino acid complexes and reduced translational efficiency [27].

Finally, after GeneDesign has been used to generate one or more condition-specific codon optimized sequences, the corresponding DNA can be synthesized and introduced into the cellular host of interest. Functional assays can be used to determine the highest performing variant.

\section{Generation of condition-specific codon bias matrices for \\ S. cerevisiae}

In order to validate our hypothesis and this approach, we selected two heterologous genes of interest, applied the approach as outlined above, expressed the resulting optimized genes in S. cerevisiae, and measured resulting protein activity. For comparison, we generated a control table and codon context matrix (hereafter referred to as the control matrix), which were assembled using the protein coding sequences of $6,666 \mathrm{~S}$. cerevisiae genes. The control table is identical to the CUTG from GenBank 
for $S$. cerevisiae, which is used commercially. A colored representation of the control matrix is shown in Figure $1 \mathrm{~b}$ (see Additional file 3 for full data set) with the $y$-axis representing the first codon and the $\mathrm{x}$-axis representing the second codon in a pair. Each square represents the probability of a codon pair occurring given that the first codon is specified, with the color blue representing a probability of one and red representing a probability of zero. The two solid blue columns correspond to a second codon for methionine (ATG) or tryptophan (TGG), both of which have no synonymous codons and therefore have a probability of 1 . Amongst the synonymous codons however, there is variation between the columns, indicating an overall genomic preference for particular synonymous codons. There is very little variation between rows, indicating that the choice in second codon is not influenced much by the choice in first codon. Since this control matrix incorporates codon context for nearly all protein coding genes in S. cerevisiae, the probability values are an average of codon usage across the entire genome. As a result, the columns become indicative of the frequency of each codon, with the rarest indicated in red.

In contrast, the matrices made for the subset of highest expressed genes and genes expressed in stationary phase (Figures 1c-1d, respectively) have significant variation between rows (see Additional file 3 for full data set). In order to compare the difference between the control matrix and condition-specific matrices we quantified the drift using the Frobenius matrix norm of the difference between the matrices. This metric is well established as a quantitative tool to determine drift between matrices of the same size [34]. A smaller Frobenius matrix norm indicates higher similarity between matrices, such that identical matrices have a norm of zero. We first evaluated the drift between the control matrix and sets of randomly selected genes as a control. Random sets of 30, 50, 100, 150, 200, 250 and 300 genes were selected in five independent events and the average matrix norm was calculated (Figure 1e). As the number of randomly considered genes increases, the Frobenius matrix norm or drift relative to the control condition decreases in a power regression fashion (of the form $y=A x^{b}$ ). This behavior is expected, as the inclusion of more genes in a codon usage matrix will result in an averaging effect that begins to resemble the control matrix composed of all gene sequences. Next, we calculated the drift between the control matrix and the highest expressed genes in S. cerevisiae [35]. The higher drift demonstrates that these genes exhibit a vastly different codon usage than the control matrix. The difference in codon usage between highly expressed genes and the rest of the genome has been previously noted [16], suggesting evolutionary pressure on codon usage. This further supports the use of only a subset of genes for determining codon usage bias.

\section{Condition-specific optimization of eGFP for high} expression outperforms wild-type and control variants

Initially, we sought to investigate the importance of condition-specific codon optimization by re-coding an $E$. coli fluorescence protein for high, constitutive expression in yeast. To do so, we established a CUB based on the 100 most highly expressed genes during growth in YPD media [35]. The associated condition-specific table and matrix were assembled as described above (Figure 1c, see Additional files 3 and 4 for full matrices and tables, respectively). An E. coli optimized green fluorescent protein $(e G F P)$ was selected as a reporter protein as it is poorly translated in yeast. Eight sequence variants of $e G F P$ were generated and compared to the wild-type sequence. One variant was optimized using the control table, one variant using the high expression table, three variants using probabilistic design based on the control matrix and three variants using probabilistic design based on the high expression matrix. The sequences for each of these variants can be found in Additional file 4. Each variant, including wild-type $e G F P$, was inserted into the $\mathrm{p} 41 \mathrm{~K}-\mathrm{GPD}$ yeast expression vector and transformed into S. cerevisiae BY4741 [36] and fluorescence was screened in mid-log phase of YPD growth using biological triplicates. We observed that, on average, the eGFP variants generated using the high expression matrix are statistically better expressed than those variants generated using the control matrix $(p$-value $=6.1 \mathrm{e}-6)$ and better than the wild-type $e G F P(\mathrm{p}$-value $=1.4 \mathrm{e}-6)($ Figure 2$)$. While all three of the high expression matrix-generated variants outperform the wild-type eGFP, only two of the three control matrix-generated variants outperform wild-type $e G F P$. These results demonstrate that optimizing codon usage specifically for high expression was effective and that the probabilistic design is suitable for generating functional variants.

We also tested the eGFP variants generated using the control and high expression table rather than a probabilistic matrix-based design. While there was no statistical difference between the two conditions, expression of these variants were higher than any of the matrixgenerated conditions. This result is not surprising given the short length of the gene (251 amino acids). However, a table-optimized approach lacks codon diversity which may ultimately become a bottleneck and decrease enzyme fitness for large metabolic engineering endeavors with multiple genes $[23,27,37]$. The fact that two tableoptimized genes were similar is not unexpected either, as there was a difference between the most abundant codon for only six of the 20 amino acids in the two tables, and thus the genes codon optimized by both tables were highly similar on a sequence basis. Nevertheless, these results illustrate that the probabilistic method used to choose codons from the condition specific matrix 


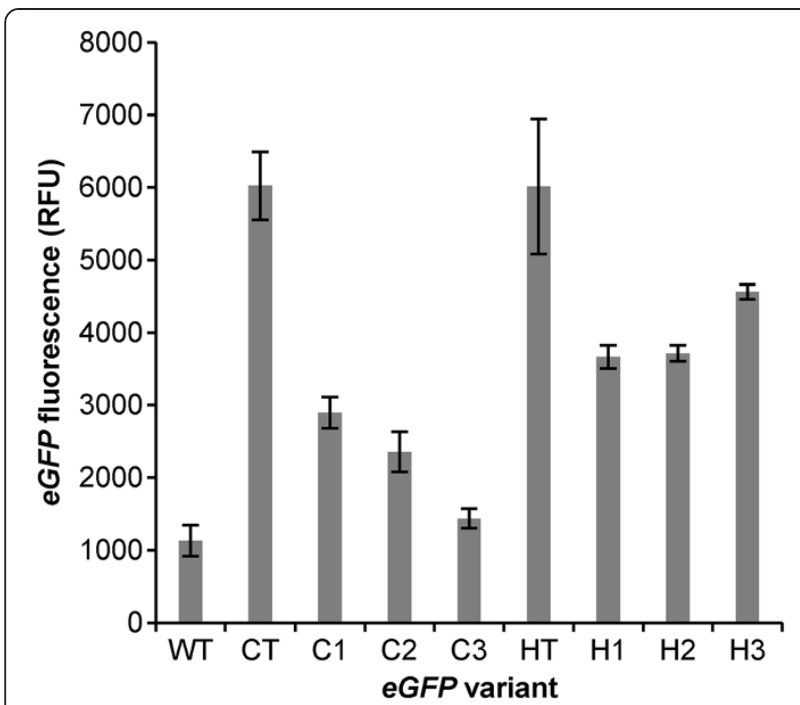

Figure 2 Optimization for a high expression condition results in eGFP expression exceeding the wild-type. In addition to wild-type eGFP, eight variants were generated. The high expression variants were made from a codon usage table $(\mathrm{HT})$ and matrix ( $\mathrm{H} 1-\mathrm{H} 3)$ constructed using the 100 most highly expressed genes in yeast grown in rich media. Control variants were constructed from the standard usage table (CT) and control matrix (C1-C3). eGFP protein expression was measured using flow cytometry for yeast grown in YPD. Biological triplicates were used to calculate standard deviations, indicated by error bars and p-values were calculated using a t-test to determine statistical significance (described in text of paper).

gives better results than that from the control matrix. As such, this is the first proof-of-concept demonstrating that using condition-specific data can improve codon optimization gene design over the traditional CUB methods.

\section{High expression and stationary phase optimization of CatA}

Next, we sought to codon optimize a catechol 1,2-dioxygenase gene (CatA) from Acinetobacter baylyi in S. cerevisiae to enable the production of muconic acid, a useful polymer precursor, in the stationary phase. This condition was chosen as it is often desirable to delay gene expression until stationary phase to increase product output and separate growth and production phases. To optimize under these conditions, a codon usage matrix was calculated using the 50 genes most differentially upregulated when comparing three day cultures to exponential growth [38] (Figure 1d, see Additional file 3 for full matrices). This data was downloaded from a previous study available under the GEO reference E-TABM-496. We designed three CatA variants using a probabilistic design based on the stationary phase matrix (named stationary \#1 through \#3), three similarly designed using the control matrix (named control \#1 through \#3), and two using the high expression matrix used above for eGFP (named high expression \#1 through \#2). Finally, we included wild-type A. baylyi CatA and a variant that was codon-optimized for expression in S. cerevisiae by Blue Heron Biotechnology using traditional codon optimization methods (referred to as the Blue Heron variant). These sequences are included in Additional file 4. Expression of these ten variants was determined using a previously described protein activity assay [22] during various stages of growth $(6,18$ and 24 hours post inoculum). We calculated the catalytic rate, $V_{\max }\left(\mathrm{mM} / \mathrm{min}^{*} \mu \mathrm{g}\right.$ protein), for each variant (Figure 3a).

In exponential phase (6 hours of growth), the $V_{\max }$ for wild-type CatA is significantly higher than the Blue Heron variant $(p$-value $=0.002)$. This illustrates again that traditional codon optimization approaches can often result in poor performance. The most highly expressed variant after 6 hours of growth is high expression \#1 whereas the lowest expressed variant is stationary \#1.

Toward late exponential phase and early stationary phase (18 hours), the CatA expression pattern shifts for each variant. Compared to the 6 hour time-point, the cellular catalytic level $\left(\mathrm{V}_{\max }\right)$ at 18 hours decreases for many of the constructs including the control variants \#1 and $\# 2$, high expression \#1, and the Blue Heron variant. By comparison, $V_{\max }$ for stationary $\# 2$ and \#3 is unchanged between 6 and 18 hours, and for stationary \#1, $\mathrm{V}_{\text {max }}$ actually increases. At 18 hours, the average $\mathrm{V}_{\max }$ for the stationary variants is significantly higher than the average for the control variants $(p$-value $=0.013)$ and the Blue Heron variant ( $\mathrm{p}$-value $=0.026$ ). While the stationary phase variants either maintain or increase their $\mathrm{V}_{\max }$, the activity of the control variants decreases significantly. Moreover, at this timepoint, the control variants perform worse than the $A$. baylyi wild-type gene.

The disparity between the average $V_{\max }$ for the stationary and control variants is even more significant ( $\mathrm{p}$-value $=$ 4.7e-4) at 24 hours. Furthermore, outside of high expression variant \#1, the stationary variants \#1 and \#3 demonstrate the highest $V_{\max }$ values. The profile for stationary \#1 is particularly interesting as it was among the worst during exponential phase and among the best at stationary phase. These results provide further demonstration that codon optimization based on condition-specific CUBs can outperform traditional approaches. By doing so, we were able to design three CatA variants which outperformed both a commercially-optimized sequence (Blue Heron variant) and three control CatA variants in stationary phase.

We have previously demonstrated that catalytic capacity is just as important as catalytic rate for the CatA enzyme [22]. To measure this, cultures with each variant were grown for 18 hours and then spiked with $1 \mathrm{mg} / \mathrm{ml}$ catechol. Resulting muconic acid (normalized to cell count) was measured via HPLC for each of the variants 

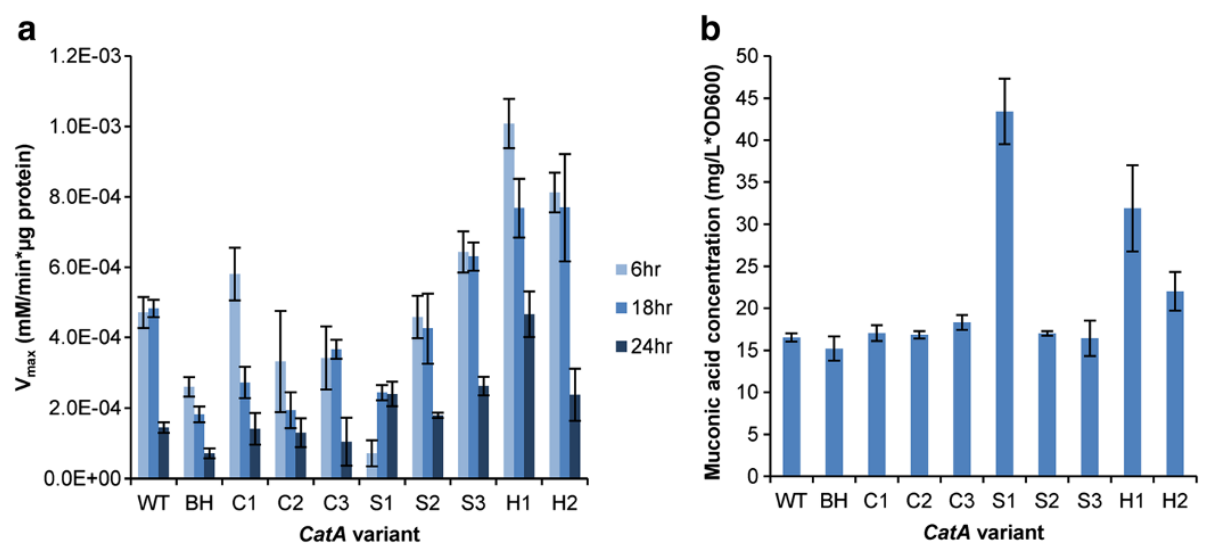

Figure 3 Optimization for stationary phase results in CatA variants that are improved at late growth. Ten CatA variants were generated, including wild-type and a version optimized by Blue Heron Biotechnology. The three stationary phase variants (S1-S3) were made from a codon usage matrix constructed using the 50 most highly expressed genes after three days of growth (see Supplementary Matrices). The two high-expression variants $(\mathrm{H} 1-\mathrm{H} 2)$ were made from a codon usage matrix constructed using the 100 most highly expressed genes in yeast grown in rich media. The three control variants (C1-C3) were constructed from the control matrix). Two assays were conducted to measure activity. $\mathbf{a}$. Cells expressing the CatA variants were grown for 6,18 or 24 hours prior to bulk protein extraction. The $V_{\max }\left(\mathrm{mM} / \mathrm{min}{ }^{*} \mu \mathrm{g}\right.$ protein) for conversion of catechol to muconic acid was determined for the bulk protein. Biological triplicates and technical triplicates were measured to determine standard deviations. b. Cells expressing the CatA variants were grown for 18 hours in $30 \mathrm{~mL}$ before spiking the media with $1 \mathrm{~g} / \mathrm{L}$ of catechol. After 24 additional hours of growth, $1 \mathrm{~mL}$ of supernatant was extracted and analyzed using HPLC, as previously described [22], to determine total muconic acid production. Normalized muconic acid levels $\left(\mathrm{mg} / \mathrm{L}^{*} \mathrm{OD}_{600}\right)$ are reported and standard deviation was determined using biological triplicates and p-values were calculated using a t-test to determine statistical significance (described in text of paper).

(Figure 3b). In this assay, the stationary \#1 variant outperformed all other variants, including the high expression \#1 variant $(p$-value $=0.036)$. Overall, this variant had a catalytic capacity that was 2.6 -fold higher than the wild-type version and nearly 2.9-fold higher than the Blue Heron optimized version in the stationary phasethe condition used to optimize this gene. These results highlight the importance and potential of condition specific codon optimization, and demonstrate for the first time that codon optimization can be used to control translation for specific environmental conditions.

\section{Analysis of transcription factor-regulated genes suggests codon usage is linked to gene regulation}

Based on the utility of condition-specific CUBs, we were interested in exploring the potential link between transcriptional and translational regulation. Utilizing a systems biology perspective, we concentrated on key global transcription factors in yeast. Specifically, we sought to calculate the CUB matrices for genes regulated by sixteen global transcription factors for S. cerevisiae: Cbflp, Dal82p, Gcn4p, Gln3p, Hap4p, Hsf1p, Leu3p, Mbp1p, Msn4p, Nrg1p, Pho4p, Rtg3p, Skn7p, Ste12p, Tec1p, and Upc2p. Codon usage matrices for the gene targets regulated by each of these global regulators were compared with the control matrix derived from all 6,666 protein coding genes (see Additional file 3 for all matrices). The Frobenius matrix norm was calculated for all pairwise combinations of the 17 matrices (control and 16 transcription factors) and this data set was used to generate a colored table, with darker colored cells representing a higher Frobenius matrix norm, and thus more distinct matrices (Figure 4).

The matrices for Rtg3p targets and Cbf1p targets are both most similar to the control matrix whereas Gcn $4 p$ and Hsflp targets are most dissimilar in their codon usage compared with the control dataset. In general, Figure 4 clearly shows that the genetic targets of transcription factors have very disparate codon usage compared to the aggregate of coding regions. Furthermore, each transcription factor matrix is more similar to the control condition than to another transcription factor matrix. This result further supports the averaging effect seen by using all protein coding genes to create CUB tables, as opposed to a subset. The fact that distinct patterns in codon usage appear for each transcription factor suggests that genetic regulation has co-evolved with codon usage. This finding supports the central hypothesis of this work - that transcriptional profiles (in the form of gene expression data) can be used to predict optimization schemes for translational regulation (in the form of codon optimization).

\section{Conclusions}

Here, we demonstrate the utility of a condition-specific codon optimization method that utilizes both systemslevel information and codon context to generate a set of potential variants using a probabilistic algorithm. While we demonstrated the effectiveness of this algorithm using 


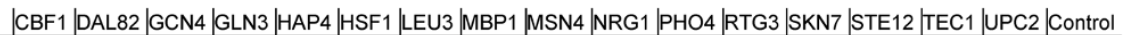

\begin{tabular}{l|r|r|r|r|r|r|r|r|r|r|r|r|r|r|r|r|r|r|r|r|}
\hline CBF1 & 0 & 7.83 & 10.3 & 5.09 & 6.92 & 10.16 & 9.58 & 6.65 & 6.78 & 5.35 & 9.04 & 4.26 & 4.94 & 7.68 & 7.21 & 6.48 & 3.53 \\
\hline DAL82 & & 0 & 11.94 & 8.23 & 9.48 & 12.1 & 11.71 & 9.08 & 9.32 & 8.03 & 11.41 & 7.79 & 7.91 & 10.01 & 9.69 & 9.59 & 7.43 \\
\hline GCN4 & & & 0 & 10.45 & 11.28 & 12.88 & 12.78 & 11.29 & 10.79 & 10.47 & 12.46 & 10.06 & 10.44 & 11.96 & 11.81 & 11.6 & 9.62 \\
\hline GLN3 & & & & 0 & 7.39 & 10.54 & 10.3 & 7.47 & 7.41 & 6.32 & 9.66 & 4.99 & 5.75 & 8.2 & 8.23 & 7.51 & 4.78 \\
\hline HAP4 & & & & & 0 & 10.81 & 11.08 & 8.53 & 8.75 & 7.39 & 10.15 & 6.54 & 7.33 & 9.04 & 8.7 & 8.54 & 5.97 \\
\hline HSF1 & & & & & & 0 & 12.66 & 11.37 & 10.86 & 10.74 & 12.77 & 10.08 & 10.23 & 11.37 & 11.54 & 11.53 & 9.51 \\
\hline LEU3 & & & & & & 0 & 10.81 & 10.54 & 10.1 & 12.5 & 9.46 & 9.84 & 11.6 & 11.05 & 10.72 & 9.08 \\
\hline MBP1 & & & & & & & & 0 & 8.12 & 7.26 & 10.55 & 6.74 & 6.97 & 9.05 & 8.58 & 8.21 & 6.21 \\
\hline MSN4 & & & & & & & & & 0 & 7.12 & 10.31 & 6.59 & 7.16 & 9.44 & 8.93 & 8.47 & 6.39 \\
\hline NRG1 & & & & & & & & & 0 & 9.19 & 5.13 & 5.8 & 8.1 & 7.98 & 7.16 & 4.37 \\
\hline PHO4 & & & & & & & & & & 0 & 9.14 & 9.59 & 10.94 & 10.72 & 10.21 & 8.66 \\
\hline RTG3 & & & & & & & & & & & & & 4.98 & 7.58 & 7.22 & 6.4 & 3.34 \\
\hline SKN7 & & & & & & & & & & & & 0 & 8.14 & 7.71 & 7.32 & 4.4 \\
\hline STE12 & & & & & & & & & & & & & & 8.64 & 9.04 & 6.9 \\
\hline TEC1 & & & & & & & & & & & & & & 0 & 8.57 & 6.8 \\
\hline UPC2 & & & & & & & & & & & & & & & 0 & 6.07 \\
\hline Control & & & & & & & & & & & & & & & & & 0
\end{tabular}

Figure 4 Drift of transcription-factor codon matrices reveals diverse codon usage relative to the control matrix. The genetic interaction targets for sixteen S. cerevisiae transcription factors were identified using the Saccharomyces Genome Database (yeastgenome.org). Using those corresponding gene target sequences, codon usage matrices were constructed for each transcription factor. Frobenius matrix norms were calculated for all matrix pairs, including the control matrix (Figure 1b) using MATLAB. The Frobenius norms represent drift between matrices and darker colored cells represent higher drift. A value of zero means the matrices are identical.

yeast and microarray data, the approach should be generic and easily adaptable to other hosts and other highthroughput datasets. While the consideration of codon pair bias and probabilistic design to create variants has been demonstrated previously [30,32], this is the first example to demonstrate that gene expression data can be used to generate high-expressing variants for a specific condition. In the case of the CatA gene, the S1 variant was high-expressing in stationary phase and low-expressing in exponential phase, resulting in the highest productivity of muconic acid of all of the variants. This is of considerable interest as it offers a previously unidentified method of control at the translational regulation level for heterologous gene expression.

It should also be noted that this is the first work to study a specific codon optimization strategy in S. cerevisiae in depth. Previous codon optimization strategies have been primarily validated using prokaryotes [3,11,30,37], endogenous expression data $[14,15,17]$, or limited heterologous gene variants $[7,39]$. In contrast, we expressed two heterologous genes in S. cerevisiae, eGFP and CatA, which were each optimized for different growth conditions. In each case, we observe improved protein expression using the condition-specific optimization technique outlined here. Specifically, eGFP expression was successfully optimized for high expression in rich media, and the CatA enzyme was optimized for high expression in stationary phase growth, with the highest expression variant resulting in 2.9-fold higher product yield over a commercially optimized variant. Finally, we demonstrated that transcriptional regulation is indeed linked to translational efficiency through the significantly different codon utilization patterns of distinctly regulated genes.
Codon optimization is an important synthetic biology tool that enables recombinant DNA expression. The algorithm we define here is simple to execute and host agnostic. As our ability and desire to produce chemicals in a renewable and environmentally-friendly capacity increases $[40,41]$, the necessity for optimized heterologous expression will likewise increase. Furthermore, many of these processes will be carried out under diverse, nonstandard environmental conditions, including changes in temperature, $\mathrm{pH}$, mineral concentration, carbon source, oxygen level, and cell growth phase. The conditionspecific approach to codon optimization described here will be a key tool to identify gene variants with the ideal codon usage for any particular condition.

\section{Methods}

\section{Microarray data analysis}

Codon usage profiles were assembled using publicly available microarray data, downloaded from the Gene Expression Omnibus (http://www.ncbi.nlm.nih.gov/geo/). Data pre-processing and normalization was performed using the Robust Multichip Average algorithm [42-44], and Bioconductor's Affy package in R version 2.15.1. Differentially expressed genes were identified using the Linear Models for Microarray Data (LIMMA) package. Probe sets were matched with $S$. cerevisiae genes using information included in Affymetrix's Expression Console Software. Genes with an adjusted p-value less than 0.05 and a logfold change greater than one or less than negative one were considered differentially expressed. A subset of differentially expressed genes (typically 50) was used to generate a condition-specific codon usage table and matrix, as described in the Results and discussion. 


\section{Plasmid construction}

Yeast expression vectors were propagated in Escherichia coli. All experiments were conducted using S. cerevisiae strain BY4741. The sequences of all genes used in this study are available in Additional file 4. The wild-type and Blue Heron Biotechnology-optimized CatA variants were taken from a previous study [22]. All other CatA variants were assembled using IDT's gBlocks. The ten Cat $A$ sequences were assembled in the p413-TEF vector [45]. The wild-type eGFP gene was amplified from the pZE-eGFP [46] plasmid using primers TAAAACACCAG AACTTAGTTTCGACGGATTCTAGAATGCGTAAAG GAGAAGAACTTTTCA and AGGTCGACGGTATCGA TAAGCTTGATATCGAATTCTTAAACTGCTGCAGCG TAGTTTTCG. The other eight $e G F P$ variants were assembled using IDT's gBlocks. The eGFP genes were cloned into the p41K-GPD plasmid [36] using yeast homologous recombination and overlapping sequences and a high efficiency, lithium-acetate transformation. The formation of correct plasmids was confirmed using DNA sequencing. For each variant, three biological replicates were isolated and stored.

\section{Growth and media conditions}

YPD media contains $20 \mathrm{~g} / \mathrm{L}$ yeast extract, $10 \mathrm{~g} / \mathrm{L}$ peptone and $10 \mathrm{~g} / \mathrm{L}$ glucose. Minimal media contains $6.7 \mathrm{~g} / \mathrm{L}$ nitrogen base, $20 \mathrm{~g} / \mathrm{L}$ glucose. Minimal media was supplemented with amino acids; $0.77 \mathrm{~g} / \mathrm{L}$ of CSM -His (MP Biomedicals) for p413 vectors and $0.79 \mathrm{~g} / \mathrm{L}$ of CSM for $\mathrm{p} 41 \mathrm{~K}$ vectors. Media for $\mathrm{p} 41 \mathrm{~K}$ vectors was supplemented with $200 \mu \mathrm{g} / \mathrm{L}$ of G418. Bacteria were grown in lysogeny broth with ampicillin. All yeast strains were grown at $30^{\circ} \mathrm{C}$ and bacteria at $37^{\circ} \mathrm{C}$. Agar plates were grown in standing incubators and cultures in shakers operating at $225 \mathrm{rpm}$.

\section{Flow cytometry}

Flow cytometry was used to determine eGFP expression. Stationary phase culture was used to inoculate $6 \mathrm{~mL}$ of YPD at an OD600 of 0.005 . Cells were grown for 12 hours, allowing cultures to reach mid-log phase. Cells were pelleted and re-suspended in cold water. Fluorescent expression profiles were determined using a FACS Fortessa. Forward scattering had a voltage setting of 209 and ampgain of 1.00, side scattering a voltage of 209 and ampgain of 1.00 and fluorescence a voltage of 308 and ampgain of 1.00. Forward and side scattering data were linear and fluorescence was collected on a logarithmic scale. Threshold was set to a forward scattering value of 5000 with an OrOperator and area scaling of 0.71. Gating and statistical analysis of the data was performed using FlowJo 7.6.

\section{Cat $A$ activity assay}

Yeast minimal media was inoculated at an OD600 of 0.1 using stationary phase cultures of the CatA variants.
Flasks contained $200 \mathrm{~mL}, 100 \mathrm{~mL}$ and $50 \mathrm{~mL}$ of media for the 6, 18 and 24 hour growth experiments respectively. After the designated time period, cells were pelleted and protein was extracted as previously described [22]. Total protein was determined using a Bradford assay. $\mathrm{V}_{\max }$ values were measured on a microgram of protein basis using a kinetic assay measuring the conversion of added catechol to muconic acid, which can be detected at $288 \mathrm{~nm}$. All biological replicates were included and measurements were done in technical triplicate. Catechol was mixed with protein extract at four concentrations, 0.1, 0.2, 0.3 and 0.4 mM, and Lineweaver-Burke plots were used to calculate $\mathrm{V}_{\max }$ in units of $\mathrm{mM} / \mathrm{min}^{*} \mu$ g protein. A higher $\mathrm{V}_{\max }$ corresponds to more CatA enzyme in the protein extract.

\section{Muconic acid production}

High pressure liquid chromatography (HPLC) was used to measure the intercellular conversion of catechol to muconic acid in S. cerevisiae cultures as previously described [22]. Triplicate yeast cultures expressing each Cat $A$ variant were grown in $30 \mathrm{~mL}$ of media for 18 hours with a starting OD600nm of 0.1. After 18 hours, cultures were spiked with $1 \mathrm{mg} / \mathrm{mL}$ of catechol and grown for an additional 24 hours. At this point, $1 \mathrm{~mL}$ of supernatant was filtered and analyzed using a Zorbax SB-Aq column (Agilent Technologies). The injection volume was $2.0 \mu \mathrm{L}$ and the mobile phase was $84 \% 25 \mathrm{mM}$ potassium phosphate buffer $(\mathrm{pH}=2.0)$ and $16 \%$ acetonitrile with a flow rate of $1.0 \mathrm{~mL} / \mathrm{min}$. The column was maintained at $30^{\circ} \mathrm{C}$ and the UV-vis absorption was measured at $280 \mathrm{~nm}$. Muconic acid production levels were calculated using a standard curve. Cis,cis-muconic acid standards were purchased from Sigma-Aldrich and cis,trans-muconic acid was provided by Draths Corporation.

\section{Matrix drift analysis}

The Frobenius matrix norm is defined as the square root of the product of the trace of the conjugate transpose of the matrix and the matrix itself:

$$
\|A\|_{F}=\sqrt{\sum_{i-1}^{m} \sum_{j=1}^{n}\left|a_{i j}\right|^{2}}=\sqrt{\operatorname{trace}(A * A)}=\sqrt{\sum_{i=1}^{\min \{m, n\}} \sigma i^{2}}
$$

The drift between any two codon usage matrices was determined by taking the difference between the matrices (excluding stop codon usage) and the Frobenius matrix norm of that resultant matrix of differences, or $\|\mathrm{A}-\mathrm{B}\|_{\mathrm{F}}$ where $\mathrm{A}$ and $\mathrm{B}$ represent two distinct matrices of identical size.

The genetic interaction targets for sixteen S. cerevisiae transcription factors were identified using the Saccharomyces Genome Database (yeastgenome.org). Using those 
corresponding gene target sequences, codon usage matrices were constructed for each transcription factor. Frobenius matrix norms were calculated for all matrix pairs, including the control matrix using MATLAB.

\section{Additional files}

\section{Additional file 1: Python script 'CodonUsageAnalysis' to read gene sequence(s) as a text file and determines the codon distribution, which is output in a .txt file.}

Additional file 2: Python script 'GeneDesign' Python script to read a protein sequence and a specified codon distribution, using this information to stochastically construct a corresponding gene sequence.

Additional file 3: Color-coded $64 \times 64$ codon usage matrices for various conditions discussed throughout the manuscript.

Additional file 4: Gene sequences, supplementary codon distribution tables.

\section{Competing interests}

The authors declare that they have no competing interests.

\section{Authors' contributions}

AML helped conceive the study, wrote python code, participated in cloning genetic constructs, participated in experiments and drafted the manuscript. KAC participated in experiments and helped draft and edit the manuscript. LGR participated in cloning genetic constructs. HAS helped conceive the study, participated in design and coordination and helped edit the manuscript. All authors read and approved the final manuscript.

\section{Acknowledgements}

We thank John Leavitt for help optimizing the muconic acid assay methods and Allison Wagman for help performing the assays. We acknowledge the Rochelle group (UT Austin, Chemical Engineering) for the use of their HPLC system. We thank Dr. Taejoon Kwon (UT Austin, Marcotte lab) for R scripts used to analyze raw microarray data. Finally, we thank Joyce Ho and Yubin Park (UT Austin, IDEAL) for help with matrix drift calculations. This work was supported by a National Science Foundation Graduate Research Fellowship to K. C. and by the National Institutes of Health (grant number R01GM090221). The content is solely the responsibility of the authors and does not necessarily reflect the official views of the National Institute of General Medical Sciences or the National Institutes of Health.

\section{Author details}

${ }^{1}$ Department of Chemical Engineering, The University of Texas at Austin, 200 E Dean Keeton St. Stop C0400, Austin, TX 78712, USA. ${ }^{2}$ Institute for Cellular and Molecular Biology, The University of Texas at Austin, 2500 Speedway Avenue, Austin, TX 78712, USA. ${ }^{3}$ Current Address: Bristol-Myers Squibb, Biologics Development, 35 South Street, Hopkinton, MA 01748, USA.

Received: 3 January 2014 Accepted: 4 March 2014

Published: 17 March 2014

\section{References}

1. Gustafsson C, Govindarajan S, Minshull J: Codon bias and heterologous protein expression. Trends Biotechnol 2004, 22:346-353.

2. Young $\mathrm{E}$, Alper $\mathrm{H}$ : Synthetic biology: tools to design, build, and optimize cellular processes. J Biomed Biotechnol 2010, 2010:130781.

3. Burgess-Brown NA, Sharma S, Sobott F, Loenarz C, Oppermann U, Gileadi O: Codon optimization can improve expression of human genes in Escherichia coli: a multi-gene study. Protein Expr Purif 2008, 59:94-102.

4. Anthony JR, Anthony LC, Nowroozi F, Kwon G, Newman JD, Keasling JD: Optimization of the mevalonate-based isoprenoid biosynthetic pathway in Escherichia coli for production of the anti-malarial drug precursor amorpha-4,11-diene. Metab Eng 2009, 11:13-19.

5. Maertens B, Spriestersbach A, von Groll U, Roth U, Kubicek J, Gerrits M, Graf M, Liss M, Daubert D, Wagner R, Schafer F: Gene optimization mechanisms: a multi-gene study reveals a high success rate of full-length human proteins expressed in Escherichia coli. Protein Sci 2010, 19:1312-1326.

6. Wiedemann B, Boles E: Codon-optimized bacterial genes improve L-Arabinose fermentation in recombinant Saccharomyces cerevisiae. Appl Environ Microbio/ 2008, 74:2043-2050.

7. Kotula L, Curtis PJ: Evaluation of foreign gene codon optimization in yeast: expression of a mouse IG kappa chain. Biotechnology (N Y) 1991 9:1386-1389.

8. Partow S, Siewers V, Daviet L, Schalk M, Nielsen J: Reconstruction and evaluation of the synthetic bacterial MEP pathway in Saccharomyces cerevisiae. PLoS One 2012, 7:e52498.

9. Hershberg R, Petrov DA: General rules for optimal codon choice. PLoS Genet 2009, 5:e1000556.

10. Plotkin JB, Kudla G: Synonymous but not the same: the causes and consequences of codon bias. Nat Rev Genet 2011, 12:32-42.

11. Fox JM, Erill I: Relative codon adaptation: a generic codon bias index for prediction of gene expression. DNA Res 2010, 17:185-196.

12. Tuller $T$, Waldman YY, Kupiec M, Ruppin E: Translation efficiency is determined by both codon bias and folding energy. Proc Natl Acad SCi U S A 2010, 107:3645-3650.

13. Nakamura $Y$, Gojobori T, Ikemura T: Codon usage tabulated from international DNA sequence databases: status for the year 2000. Nucleic Acids Res 2000, 28:292.

14. Jansen R, Bussemaker HJ, Gerstein M: Revisiting the codon adaptation index from a whole-genome perspective: analyzing the relationship between gene expression and codon occurrence in yeast using a variety of models. Nucleic Acids Res 2003, 31:2242-2251.

15. Sharp PM, Li WH: The codon adaptation index-a measure of directional synonymous codon usage bias, and its potential applications. Nucleic Acids Res 1987, 15:1281-1295.

16. Sharp PM, Tuohy TM, Mosurski KR: Codon usage in yeast: cluster analysis clearly differentiates highly and lowly expressed genes. Nucleic Acids Res 1986, 14:5125-5143.

17. Wright F: The 'effective number of codons' used in a gene. Gene 1990, 87:23-29.

18. Puigbo P, Guzman E, Romeu A, Garcia-Vallve S: OPTIMIZER: a web server for optimizing the codon usage of DNA sequences. Nucleic Acids Res 2007, 35:W126-W131.

19. Puigbo P, Bravo IG, Garcia-Vallve S: E-CAl: a novel server to estimate an expected value of Codon Adaptation Index (eCAl). BMC Bioinformatics 2008, 9:65.

20. Wang TT, Cheng WC, Lee BH: A simple program to calculate codon bias index. Mol Biotechnol 1998, 10:103-106.

21. Alexeyev MF, Winkler HH: Gene synthesis, bacterial expression and purification of the Rickettsia prowazekii ATP/ADP translocase. Biochim Biophys Acta 1999, 1419:299-306.

22. Curran KA, Leavitt JM, Karim AS, Alper HS: Metabolic engineering of muconic acid production in Saccharomyces cerevisiae. Metab Eng 2013, 15:55-66.

23. Agashe D, Martinez-Gomez NC, Drummond DA, Marx CJ: Good codons, Bad transcript: large reductions in gene expression and fitness arising from synonymous mutations in a Key enzyme. Mol Biol Evol 2013, 30(3):549-560. doi:10.1093/molbev/mss273.

24. Forster AC: Synthetic biology challenges long-held hypotheses in translation, codon bias and transcription. Biotechnol J 2012, 7:835-845.

25. Dong H, Nilsson L, Kurland CG: Co-variation of tRNA abundance and codon usage in Escherichia coli at different growth rates. J Mol Biol 1996, 260:649-663.

26. Frenkel-Morgenstern M, Danon T, Christian T, Igarashi T, Cohen L, Hou YM, Jensen $\sqcup$ : Genes adopt non-optimal codon usage to generate cell cycle-dependent oscillations in protein levels. Mol Syst Biol 2012, 8:572.

27. Gingold H, Pilpel Y: Determinants of translation efficiency and accuracy. Mol Syst Biol 2011, 7:481.

28. Hershberg R, Petrov DA: Selection on codon bias. Annu Rev Genet 2008, 42:287-299.

29. Yarus M, Folley LS: Sense codons are found in specific contexts. J Mol Biol 1985, 182:529-540.

30. Chung BK, Lee DY: Computational codon optimization of synthetic gene for protein expression. BMC Syst Biol 2012, 6:134.

31. Tats A, Tenson T, Remm M: Preferred and avoided codon pairs in three domains of life. BMC Genomics 2008, 9:463. 
32. Chung BK, Yusufi FN, Mariati, Yang Y, Lee DY: Enhanced expression of codon optimized interferon gamma in CHO cells. J Biotechnol 2013, 167:326-333.

33. Coleman JR, Papamichail D, Skiena S, Futcher B, Wimmer E, Mueller S: Virus attenuation by genome-scale changes in codon pair bias. Science 2008, 320:1784-1787.

34. Owrutsky P, Khaneja N: Control of inhomogeneous ensembles in the presence of a random periodic drift. In American Control Conference (ACC), 2012; 27-29 June 2012; 2012:5090-5095.

35. Holstege FC, Jennings EG, Wyrick JJ, Lee TI, Hengartner CJ, Green MR, Golub TR, Lander ES, Young RA: Dissecting the regulatory circuitry of a eukaryotic genome. Cell 1998, 95:717-728.

36. Karim AS, Curran KA, Alper HS: Characterization of plasmid burden and copy number in Saccharomyces cerevisiae for optimization of metabolic engineering applications. Fems Yeast Res 2013, 13:107-116.

37. Menzella HG: Comparison of two codon optimization strategies to enhance recombinant protein production in Escherichia coli. Microb Cell Fact 2011, 10:15

38. Westholm JO, Nordberg N, Muren E, Ameur A, Komorowski J, Ronne H: Combinatorial control of gene expression by the three yeast repressors Mig1, Mig2 and Mig3. BMC Genomics 2008, 9:601.

39. Norkiene M, Gedvilaite A: Influence of codon bias on heterologous production of human papillomavirus type 16 major structural protein L1 in yeast. Scientific World J 2012, 2012:979218.

40. Curran KA, Alper HS: Expanding the chemical palate of cells by combining systems biology and metabolic engineering. Metab Eng 2012, 14:289-297.

41. Krivoruchko A, Siewers V, Nielsen J: Opportunities for yeast metabolic engineering: Lessons from synthetic biology. Biotechnol J 2011, 6:262-276.

42. Irizarry RA, Bolstad BM, Collin F, Cope LM, Hobbs B, Speed TP: Summaries of affymetrix GeneChip probe level data. Nucleic Acids Res 2003, 31:e15.

43. Irizarry RA, Hobbs B, Collin F, Beazer-Barclay YD, Antonellis KJ, Scherf U, Speed TP: Exploration, normalization, and summaries of high density oligonucleotide array probe level data. Biostatistics 2003, 4:249-264.

44. Bolstad BM, Irizarry RA, Astrand M, Speed TP: A comparison of normalization methods for high density oligonucleotide array data based on variance and bias. Bioinformatics 2003, 19:185-193.

45. Mumberg D, Müller R, Funk M: Yeast vectors for the controlled expression of heterologous proteins in different genetic backgrounds. Gene 1995, 156:119-122.

46. Andersen JB, Sternberg C, Poulsen LK, Bjorn SP, Givskov M, Molin S: New unstable variants of green fluorescent protein for studies of transient gene expression in bacteria. Appl Environ Microbiol 1998, 64:2240-2246.

doi:10.1186/1752-0509-8-33

Cite this article as: Lanza et al: A condition-specific codon optimization approach for improved heterologous gene expression in Saccharomyces cerevisiae. BMC Systems Biology 2014 8:33.

\section{Submit your next manuscript to BioMed Central and take full advantage of:}

- Convenient online submission

- Thorough peer review

- No space constraints or color figure charges

- Immediate publication on acceptance

- Inclusion in PubMed, CAS, Scopus and Google Scholar

- Research which is freely available for redistribution 